\title{
New insights and therapeutic targets: Lung injury and disease
}

Brandon A. Guenthart, MD, ${ }^{\mathrm{a}, \mathrm{b}}$ Ya-Wen Chen, $\mathrm{PhD},{ }^{\mathrm{c}, \mathrm{d}, \mathrm{e}, \mathrm{f}}$ and Matthew Bacchetta, MD ${ }^{\mathrm{a}, \mathrm{g}}$

From the ${ }^{\mathrm{a}}$ Department of Biomedical Engineering, Columbia University, New York, NY; ${ }^{\mathrm{b}}$ Department of Surgery, Weill Cornell Medical Center, New York, NY; ${ }^{\mathrm{c} C}$ Columbia Center for Human Development and ${ }^{\mathrm{d}}$ Department of Medicine, Columbia University Medical Center, Columbia University, New York, NY; ${ }^{e}$ Hastings Center for Pulmonary Research, ${ }^{\mathrm{f}}$ Department of Medicine, Keck School of Medicine, University of Southern California, Los Angeles, Calif; and ${ }^{\mathrm{g}}$ Department of Thoracic Surgery, Vanderbilt University Medical Center, Nashville, Tenn.

Received for publication Aug 27, 2018; revisions received Sept 9, 2018; accepted for publication Sept 10, 2018. Address for reprints: Matthew Bacchetta, MD, Department of Surgery, Vanderbilt Medical Center, 609 Oxford House, 1313 21st Ave S, Nashville, TN 37232 (E-mail: matthew.bacchetta@vanderbilt.edu).

J Thorac Cardiovasc Surg 2019;157:416-20

$0022-5223 / \$ 36.00$

Copyright (c) 2018 by The American Association for Thoracic Surgery

https://doi.org/10.1016/j.jtcvs.2018.09.026

Feature Editor's Note-The lung is an organ that is not typically thought to be capable of regeneration; however, this is a common misconception. It has indeed been shown in both mice and humans that new lung growth can and does occur in the adult lung. Whereas most thoracic surgeons are aware of the compensatory increase in lung volume that results after lung resection (most pronounced in the contralateral lung after pneumonectomy), this phenomenon is commonly regarded simply as lung hyper-expansion, and the proliferation of lung cells with regeneration of lung architecture is often not appreciated. In the human adult, for example, lung growth is characterized by an increase in the number of alveoli, rather than enlargement of existing alveoli ( $\mathrm{N}$ Engl J Med. 2012;367:244). Two recent publications in Nature report the discovery of a novel progenitor cell in the lung, a subset of alveolar type II cells, that proliferate rapidly; differentiate into other cell types, including alveolar type I cells, and regenerate injured lung epithelium. The clinical implications of these findings for patients with end-stage lung disease are vast. Harnessing the regenerative power of lung progenitor cells, whether through in vivo targeting of these cells or through transfer of in vitro engineered alveolar epithelial type II cells, offers promising strategies for treatment of patients with acute and chronic lung injury. The following Feature Expert Opinion article offers the reader a concise introduction to this field, delivers a salient overview of this discovery, and forecasts an exciting era of clinical translation.

\section{Bryan M. Burt, MD}

The socioeconomic influence and humanitarian burden of end-stage lung disease continues to climb throughout the world. Unfortunately, a paucity of breakthroughs in the

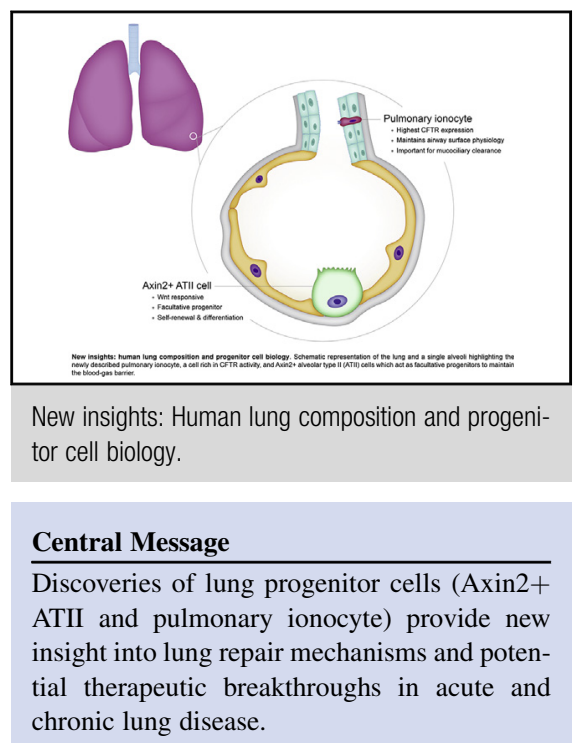

See Editorial Commentary page 421.

field has largely limited the treatment for these patients to lung transplantation, a lifesaving intervention that is highly constrained by the critical shortage of suitable donor lungs and graft durability. ${ }^{1}$ Whereas patients with heart failure have mechanical circulatory support options such as ventricular assist devices, temporizing percutaneous support devices, and total artificial hearts, patients with end-stage lung disease are left with few options. Arguably, medical management of heart disease has also drastically outpaced the medical management of chronic lung disease with the exception of recent advances in the treatment of cystic fibrosis and pulmonary arterial hypertension. New methods are needed to recover and regenerate lung tissue to address the critical shortage of donor organs and to develop novel therapies to treat lung disease.

Regenerative strategies have broad applications in the repair of injured donor lungs (lungs that are currently being discarded), and ex vivo lung perfusion (EVLP) has significantly contributed to our understanding of donor lung injury. ${ }^{2,3}$ However, the clinical influence and utility of EVLP remains limited despite progress in the management of marginal donor organs and there is no evidence to suggest that EVLP systems provide a mechanism for organ regeneration. Many investigators have used decellularization/recellularization and bioreactor platforms to demonstrate a pathway to organ regeneration, but 

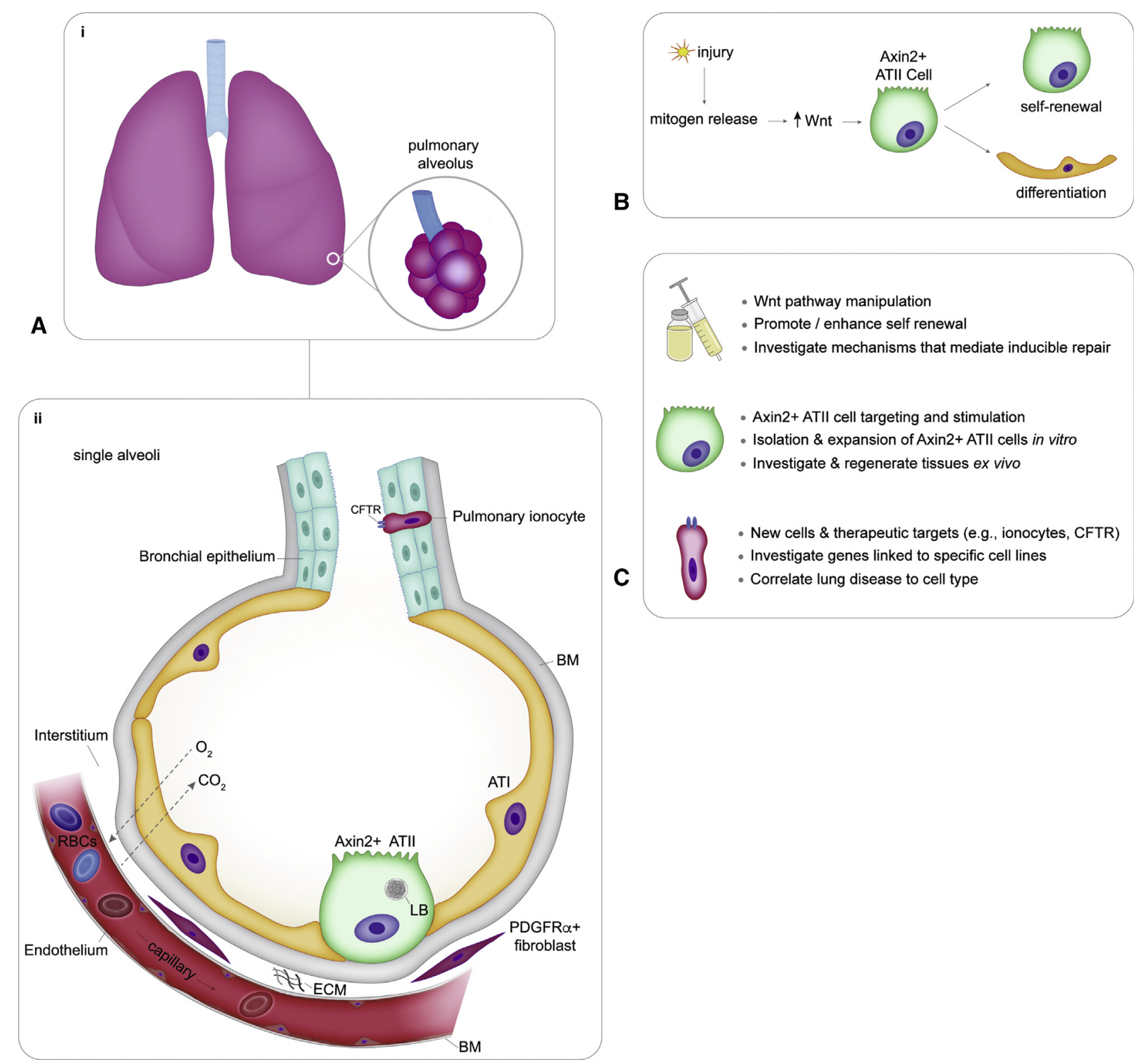

FIGURE 1. Alveolar composition, microenvironoment, and targets for regenerative medicine. A, Schematic representation of lung structure and regional composition. (i) Lungs displayed on a macro level down to the distal respiratory zone and alevolar ducts. (ii) A single alveoli with 2 epithelial cell types forming the distal gas exchange interface. Thin alveolar epithelial cells (alveolar epithelial cell type I [ATIs]) and cuboidal alveolar epithelial cells (alveolar epithelial cell type II [ATII]). The local niche supporting and influencing the Axin2+ ATII cell population includes surrounding cells (ie, ATIs, fibroblasts, and vascular endothelial cells) and interstitial components, including extracellular matrix proteins. Also shown is the newly described pulmonary ionocyte, a cell rich in cystic fibrosis transmembrane conductance regulator $(C F T R)$ activity. B, Mechanism of ATII cells acting as facultative progenitors. The process begins with lung injury resulting in cell death leading to the release of mitogens. Surviving Axin2+ ATII cells upregulate the Wnt pathway in an autocrine fashion, or respond to proliferative signals released by surrounding PDGFRa + fibroblasts. ATII cells can self-renew and produce new ATII cells or differentiate and produce ATI cells. C, Possible therapeutic targets and regenerative strategies. Categories include targeted pharmacotherapies, use of endogenous progenitors, and exploring possibilities of newly discovered cellular and genetic correlates. $B M$, Basement membrane; $E C M$, extra-cellular matrix; $L B$, lamellar body (surfactant containing); RBCs, red blood cells.

convincing data for practical applications are lacking. Efforts by developmental biologists and stem cell scientists have pushed the field of regeneration forward at the microlevel, but bioengineering a functional lung graft from a fully decellularized or acellular scaffold still faces substantial hurdles.

Although considerable efforts are underway to address the critical shortage of transplantable lungs, others are 
attempting to elucidate and harness the lung's innate repair mechanisms to treat or reverse the trajectory of disease. The lung's cellular composition, with region-specific variability and function, is complex and not fully understood. It is a relatively quiescent organ with low cell turnover, and contains adult stem cells that reside in the proximal and distal airways. These cells have the ability to undergo self-renewal and give rise to different cell types. The local microenvironment plays an important role in dictating cell fate. Key questions in the field remain. What cell populations maintain the integrity of the blood-gas barrier? What key aspects of the local niche and signaling pathway guide this process? How can these endogenous progenitors and knowledge of signaling pathways be harnessed to treat lung disease or regenerate tissue? What cell lineages play a role in specific lung pathology and can these represent new therapeutic targets? Recent publications by 4 research teams offer insight and provide a framework for further investigations. ${ }^{4-7}$

\section{Lung Progenitor Cells}

Two recent publications ${ }^{4,5}$ have described a small subset of Wnt-responsive alveolar type II (ATII) cells that act as facultative progenitor cells in the distal lung. Using an H1N1 influenza injury model, Zacharias and colleagues ${ }^{4}$ performed lineage-tracing experiments to demonstrate that Axin2+ (a downstream indication of Wnt pathway activation) population undergoes significant self-renewal and generates new alveolar type I cells (Figure 1). These Axin2+ ATII cells were shown to be distinctly different from previously described Krt5+ epithelial basal cells that migrate into the distal lung in response to injury. Detailed genetic analysis revealed that Axin2+ ATII cells are genetically distinct from other ATII cells, with a profile rich in developmental genes. In organoid culture the authors showed that when TM4SF1+ (a conserved cell surface maker present in mouse and human epithelial progenitors) cells were depleted there was a dramatic loss in organoid formation, suggesting they play a role in promoting self-organization and proliferation.

Utilizing a similar mouse model and methodology, Nabhan and colleagues ${ }^{5}$ described this subset of Axin2+ ATII cells and demonstrated their ability to rapidly proliferate and regenerate injured epithelium. In their results, ${ }^{5}$ the authors describe 2 Wnt signaling pathways. In 1 pathway, juxtacrine signaling by neighboring fibroblasts (PDGFR $\alpha+$ ) enable Axin2 + ATII cells to divide intermittently (selfrenewal) and give rise to daughter alveolar type I cells (ie, transdifferentiate) when they leave the Wnt niche. In the other pathway, in response to severe injury, normally quiescent ATII cells turn on Axin2, and thus switch to autocrine control, granting the quiescent ATII cells progenitor properties. This enables rapid proliferation independent of the stromal niche. Although both groups came to similar conclusions regarding the activity of Axin2+ ATII cells, they came to a different conclusion about their prevalence. Zacharias and colleagues ${ }^{4}$ reported that approximately $20 \%$ of ATII cells in normal lung express Axin2, whereas Nabhan and colleagues ${ }^{5}$ reported that to be $1 \%$. Although variations in tamoxifen dosing used by each group during cell induction may account for this, additional investigation will be needed to elucidate this further.

These exciting results offer hope for patients with end stage lung disease. The manipulation of this population of cells in vivo (eg, targeted therapies to augment their function) may lead to breakthroughs in the treatment of acute lung injury as well as states of chronic disease. Additionally, isolating and expanding these cells in vitro may contribute to the field of tissue engineering or be used as a deliverable cell-based therapeutic agent.

\section{Cellular Composition of the Lung}

The lung is an extremely complex organ with hierarchical 3-dimensional structure, region-specific composition and function, and as many as 40 different cell types. ${ }^{10}$ This complexity poses inherent challenges to the clinician treating lung disease and the biomedical engineer attempting to recapitulate lung tissue de novo. Two independent research teams have made significant contributions to our understanding of the composition and cellular interplay of the lung's conducting airway in recent studies. ${ }^{6,7}$

Plasschaert and colleagues ${ }^{6}$ performed single-cell RNA sequencing (scRNA-seq) on thousands of human bronchial epithelial cells and mouse tracheal epithelial cells. Profiling was conducted on cells cultured using an air-liquid interface culture and from mouse tracheas following injury with polidocanol (a sclerosant) to analyze and compare cell states during homeostasis and repair. They describe an epithelial cell line with a unique gene signature not previously described. The authors found these cells to be rich in cystic fibrosis transmembrane conductance regulator (CFTR) and other chloride channels, and named these cells pulmonary ionocytes.

In a largely parallel analysis by Montoro and colleagues, the authors combined scRNA-seq and genetic lineage tracing over time to profile mouse tracheal epithelial cells. They also confirmed the presence of a new cell type, named by both groups as the pulmonary ionocyte (Figure 1). High levels of CFTR expression by pulmonary ionocytes were demonstrated using quantitative polymerase chain reaction with reverse transcription analysis. Authors also assessed mucus viscosity and ciliary beat frequency in a mouse model to demonstrate that ionocyte function is correlated with normal airway surface physiology and mucus viscosity, which is essential for effective mucociliary clearance. Lastly, to corroborate findings obtained in the mouse model, primary human airway cells were analyzed. RNA in situ hybridization confirmed the presence of ionocytes in human airways 
and scRNA-seq demonstrated that ionocytes comprise $0.5 \%$ to $1.5 \%$ of epithelial cells in the conducting airways.

Mouse models have often been challenged because differences in mouse and human epithelium are well described. ${ }^{11}$ This new methodology will allow researchers to pinpoint similarities between mouse and human airways to develop more reliable translational models. Further work to analyze gene expression in diseased lungs and to expand profiling to include the respiratory zone will add still more to our current understanding.

\section{Future Directions}

These studies have laid invaluable groundwork and provided keen insight into human lung composition and progenitor cell biology. The discovery of a new cell type that expresses more CFTR than any other airway cell, may change our current understanding and alter therapeutic strategies for patients with cystic fibrosis. Newly developed cellular analytics can now be applied to investigate the genetic profile of healthy and diseased epithelial cells and be used to link lung disease with specific cell populations. Work aimed at building an atlas of genes and cellular road map of the human airway will undoubtedly lead to future discoveries as new cells and rare cell subtypes become apparent.

Harnessing the regenerative power of endogenous cells, such as the newly discovered Axin $2+$ ATII cells, ${ }^{4,5}$ may lead to accelerated repair in the setting of acute lung injury, or be implemented as an early interventional strategy to alter the course of chronic lung disease. Although targeted disease and cell-specific therapies aimed at typically quiescent progenitor cells hold tremendous promise, they will require further investigation and studies in animal models and human tissue. Additional work is also needed to better understand how these cells work synergistically with other adult progenitor cells to maintain the integrity of airway epithelium. Because that interplay is better understood there is potential for influence and intersection with the field of induced pluripotent stem cells (iPSCs) and regenerative medicine. Undifferentiated and pluripotent cell lines have gained tremendous interest but progress has been slow due to technical challenges (eg, expansion and tumor forming potential), high cost, and regulatory stringency. The use of differentiated cells from iPSCs in coordination with, or in addition to, endogenous progenitors may prove to be an effective strategy in advancing the field.

Several endogenous progenitors, and their dynamic interplay within their niche and between mesenchymal cells, have been identified over the past few decades in steady state and following injury using mouse models. ${ }^{12-17}$ However, mouse lung differs from human lung in many aspects, and thus the actual relevance of these identified murine stem cell populations to humans is unclear. Organoids, 3-dimensional structures cultured in vitro that mimic the microanatomy and recapitulate some function of an organ, can be generated from a limited starting material, such as biopsies, or from directed differentiation of human PSCs, including embryonic stem cells and iPSCs. Previously, lung bud organoids have been successfully derived from human PSCs and displayed branching airway and early alveolar structure. ${ }^{18}$ Other lung organoids or lung sphere cultures have also been reported. ${ }^{19-22}$ Endogenous progenitors, such as the Axin2+ population, may play an important role in advancing the organoid field and open new avenues of research into biological processes (eg, repair, stem cell niche, and cell signaling) in vitro. The development of more robust human mimetic organoids could provide a platform to verify findings in animal models and lead to breakthroughs in drug testing, lung disease modeling, or become a powerful cell source for deliverable therapeutics.

Lastly, integrating the work of developmental biologists with whole organ support systems may provide a pathway to fulfill the promise within both fields to address the shortage of donor lungs. The field of tissue and organ regeneration continues to make progress in the development of bioreactors in both small and large animal models. ${ }^{23-25}$ Although several groups have developed methods of creating whole organ grafts, none have succeeded in building a fully recellularized lung that is both functional and durable. Perhaps these recent breakthroughs, and future insights gained based on their methodologies, will provide the missing fuel needed to successfully create functional lung tissue and whole organ constructs.

\section{Conflict of Interest Statement}

Authors have nothing to disclose with regard to commercial support.

\section{References}

1. Giwa S, Lewis JK, Alvarez L, Langer R, Roth AE, Church GM, et al. The promise of organ and tissue preservation to transform medicine. Nat Biotechnol. 2017; 35:530-42.

2. Cypel M, Yeung JC, Hirayama S, Rubacha M, Fischer S, Anraku M, et al. Technique for prolonged normothermic ex vivo lung perfusion. J Heart Lung Transpl. 2008;27:1319-25.

3. Cypel M, Keshavjee S. Extending the donor pool: rehabilitation of poor organs. Thorac Surg Clin. 2015;25:27-33

4. Zacharias WJ, Frank DB, Zepp JA, Morley MP, Alkhaleel FA, Kong J, et al. Regeneration of the lung alveolus by an evolutionarily conserved epithelial progenitor. Nature. 2018;555:251-5.

5. Nabhan AN, Brownfield DG, Harbury PB, Krasnow MA, Desai TJ. Single-cell Wnt signaling niches maintain stemness of alveolar type 2 cells. Science. 2018;359:1118-23

6. Plasschaert L, Žilionis R, Choo-Wing R, Savova V, Knehr J, Roma G, et al. A single-cell atlas of the airway epithelium reveals the CFTR-rich pulmonary ionocyte. Nature. 2018;560:377-81.

7. Montoro DT, Haber AL, Biton M, Vinarsky V, Lin B, Birket Se, et al. A revised airway epithelial hierarchy includes CFTR-expressing ionocytes. Nature. 2018; 560:319-24

8. Zuo W, Zhang T, Wu DZ, Guan SP, Liew AA, Yamamoto Y, et al. p63+ Krt5+ distal airway stem cells are essential for lung regeneration. Nature. 2015;517: 616-20. 
9. Vaughan AE, Brumwell AN, Xi Y, Gotts JE, Brownfield DG, Treutlein B, et al. Lineage-negative progenitors mobilize to regenerate lung epithelium after major injury. Nature. 2015;517:621-5.

10. Wagner DE, Bonvillain RW, Jensen T, Girard ED, Brunnell BA, Finch CM, et al. Can stem cells be used to generate new lungs? Ex vivo lung bioengineering with decellularized whole lung scaffolds. Respirology. 2013;18:895-911.

11. Tata PR, Rajagopal J. Plasticity in the lung: making and breaking cell identity. Development. 2017;144:755-66.

12. Kim CFB, Jackson EL, Woolfenden AE, Lawrence S, Babar I, Vogel S, et al. Identification of bronchioalveolar stem cells in normal lung and lung cancer. Cell. 2005;121:823-35.

13. Rawlins EL, Okubo T, Xue Y, Brass DM, Auten RL, Hasegawa H, et al. The role of Scgb1a1+ Clara cells in the long-term maintenance and repair of lung airway, but not alveolar, epithelium. Cell Stem Cell. 2009;4:525-34.

14. Barkauskas CE, Cronce MJ, Rackley CR, Bowie EJ, Keene DR, Stripp BR, et al. Type 2 alveolar cells are stem cells in adult lung. J Clin Invest. 2013;123: 3025-36.

15. Desai TJ, Brownfield DG, Krasnow MA. Alveolar progenitor and stem cells in lung development, renewal and cancer. Nature. 2014;507:190.

16. Rawlins EL. Stem cells: emergency back-up for lung repair. Nature. 2015;517: 556.

17. Lee J-H, Tammela T, Hofree M, Choi J, Marajanovic ND, Han S, et al. Anatomically and functionally distinct lung mesenchymal populations marked by Lgr5 and Lgr6. Cell. 2017;170:1149-63.e1112.
18. Chen Y-W, Huang SX, de Carvalho ALRT, Ho SH, Islam MN, Volpi S, et al. A three-dimensional model of human lung development and disease from pluripotent stem cells. Nature Cell Biol. 2017;19:542-9.

19. Dye BR, Hill DR, Ferguson MA, Tsai YH, Nagy MS, Dyal R, et al. In vitro generation of human pluripotent stem cell derived lung organoids. Elife. 2015;4.

20. Dye BR, Dedhia PH, Miller AJ, Nagt MS, White ES, Shea LD, et al. A bioengineered niche promotes in vivo engraftment and maturation of pluripotent stem cell derived human lung organoids. Elife. 2016;5:e19732.

21. Jacob A, Morley M, Hawkins F, McCauley KB, Jean JC, Heins H, et al. Differentiation of human pluripotent stem cells into functional lung alveolar epithelial cells. Cell Stem Cell. 2017;21:472-88.e410.

22. McCauley KB, Hawkins F, Serra M, Thomas DC, Jacob A, Kotton DN. Efficient derivation of functional human airway epithelium from pluripotent stem cells via temporal regulation of Wnt signaling. Cell Stem Cell. 2017;20: 844-57.e846.

23. Ott HC, Clippinger B, Conrad C, Schuetz C, Pomerantseva I, Ikonomou L, et al. Regeneration and orthotopic transplantation of a bioartificial lung. Nat Med. 2010;16:927-33.

24. Dorrello NV, Guenthart BA, O'Neill JD, Kim J, Cunningham K, Chen YW, et al. Functional vascularized lung grafts for lung bioengineering. Sci Adv. 2017;3: e1700521.

25. Nichols JE, La Francesca S, Niles JA, Vega SP, Argueta LB, Frank L, et al. Production and transplantation of bioengineered lung into a large-animal model. Sci Transl Med. 2018;10(452). 